\title{
Impaired Sequential Egocentric and Allocentric Memories in Forebrain-Specific-NMDA Receptor Knock-Out Mice during a New Task Dissociating Strategies of Navigation
}

\author{
Laure Rondi-Reig, ${ }^{1 \star}$ Géraldine H. Petit ${ }^{1,2 \star}$ Christine Tobin, ${ }^{2}$ Susumu Tonegawa, ${ }^{3}$ Jean Mariani, ${ }^{2}$ and Alain Berthoz ${ }^{1}$ \\ ${ }^{1}$ Collège de France, Centre National de la Recherche Scientifique (CNRS), Unité Mixte de Recherche (UMR) 7152, Laboratoire de Physiologie de la \\ Perception et de l'Action, F-75005 Paris, France, ${ }^{2}$ Université Pierre et Marie Curie-Paris 6, CNRS, UMR 7102, Laboratoire Neurobiologie des Processus \\ adaptatifs, Equipe Développement et Vieillissement du Système Nerveux, F-75005 Paris, France, and ${ }^{3}$ The Picower Institute for Learning and Memory, \\ Howard Hughes Medical Institute, Department of Biology, Massachusetts Institute of Technology, Cambridge, Massachusetts 02139
}

The hippocampus is considered to play a role in allocentric but not in egocentric spatial learning. How does this view fit with the emerging evidence that the hippocampus and possibly related cortical areas are necessary for episodic-like memory, i.e., in all situations in which events need to be spatially or sequentially organized? Are NMDA receptor-dependent mechanisms crucial for the acquisition of spatiotemporal relationships? To address this issue, we used knock-out (KO) mice lacking hippocampal CA1 NMDA receptors and presenting a reduction of these receptors in the deep cortical layers (NR1-KO mice). A new task (the starmaze) was designed, allowing us to distinguish allocentric and sequential-egocentric memories. NR1-KO mice were impaired in acquiring both types of memory. Our findings suggest that memories composed of multiple spatiotemporal events require intact NMDA receptors-dependent mechanisms in CA1 and possibly in the deep cortical layers.

Key words: hippocampus; starmaze; NMDA receptors; sequential-egocentric strategy; allocentric strategy; episodic-like memory

\section{Introduction}

Spatial navigation to a hidden goal can be supported by two different orientation systems: a place (or allocentric) system and a taxon (or egocentric) system (O’Keefe and Nadel, 1978). Existing data suggest a functional dissociation between the forebrain (including the hippocampal formation) and the basal ganglia in mediating allocentric and egocentric memory, respectively (DeCoteau and Kesner, 2000), (for review, see Packard and Knowlton, 2002; White and McDonald, 2002).

Arguments for the role of the hippocampus in allocentric learning come from convergent findings obtained using genetic manipulation, hippocampal lesions, and place cell recordings (O'Keefe and Nadel, 1978; Morris et al., 1982; Eichenbaum et al., 1990). Both pharmacological and genetic approaches have shown that hippocampal NMDA receptors (NMDARs), particularly in the CA1 region (Morris et al., 1986; Tsien et al., 1996b) but not in

Received April 7, 2005; revised Feb. 23, 2006; accepted Feb. 24, 2006.

This work was supported by Fondation pour la Recherche Médicale Grant SRI20001117030 (L.R.-R.), the Centre National d'Etudes Spatiales, GIS "Longévité" Grant L0201, and ACI Neuroscience Intégratives et Computationnelles Grant NIC 0083. This work was also supported in part by a National Institutes of Health grant to S.T. We thank V. Gautheron, P. LeBoucher, C. Maillard, M. Kollen, and C. Oualian for technical help, F. Maloumian for help with the figures, P. Bouquet for histology, P. Thouvenot for taking care of the animal facility, and A. Lohof and M. Willson for reviewing the English. We thank our colleague A. Arleo for critical reading of this manuscript.

*L.R.-R. and G.H.P. contributed equally to this work.

Correspondence should be addressed to Laure Rondi-Reig, Collège de France, Unité Mixte de Recherche 7152, Centre National de la Recherche Scientifique, Laboratoire de Physiologie de la Perception et de I'Action, 75005 Paris, France. E-mail: laure.rondi-reig@college-de-france.fr.

D01:10.1523/JNEUROSCI.3408-05.2006

Copyright $\odot 2006$ Society for Neuroscience $\quad$ 0270-6474/06/264071-11\$15.00/0 the CA3 (Lee and Kesner, 2002; Nakazawa et al., 2002, 2003), are required for the acquisition of spatial memories (for review, see Nakazawa et al., 2004).

The intact egocentric learning ability of animals with hippocampal dysfunction has been demonstrated in paradigms in which the animal must learn a specific trajectory. These tasks can be learned using what we will call a simple egocentric strategy, e.g., turning to the left in a T-maze (Packard and McGaugh, 1996) or finding a fixed hidden platform from a fixed departure point in the Morris water maze (de Bruin et al., 2001). What would happen in the case of a sequentially organized egocentric strategy, i.e., when learning the trajectory to a hidden target requires memorizing a sequence of motion directions associated with different choice points? Behavioral experiments have shown that the hippocampus plays a critical role when distinct experiences must be encoded in relation to one another and linked sequentially (Eichenbaum, 2001). Similarly, electrophysiological recordings have indicated that hippocampal neural activity seems to support spatiotemporal coding (Frank et al., 2000; Wood et al., 2000; Ferbinteanu and Shapiro, 2003) (but see Lenck-Santini et al., 2001). Nonspatial memory requiring relational and temporal coding has been shown to depend on CA1 NMDARs (Huerta et al., 2000; Rondi-Reig et al., 2001).

In addition, the deep cortical layers of both prefrontal and parietal cortex have been proposed to participate in path selection (Poucet et al., 2004). Deep cortical layers of the prefrontal cortex presented neural activities related to the choice of a direction at a specific intersection (Jung et al., 1998). Using Arc ex- 
pression, Burke et al. (2005) showed that the deep layers of posterior parietal cortex differentiate between clockwise and counterclockwise turns within the same room. These results suggest a role for neocortical areas in the encoding of information related to a specific movement.

Altogether, these findings suggest that CA1-NMDAR-dependent mechanisms, in possible interaction with cortical area mechanisms, could contribute to the acquisition of a type of memory composed of multiple episodes spatially or sequentially linked. To test this hypothesis, we used NR1-KO mice that lack hippocampal CA1-NMDARs and present a decrease of NMDARs in the deep cortical layers.

\section{Materials and Methods \\ Experimental subjects}

We used mice in which the knock-out of the gene for the NR1 subunit of the NMDA receptor is primarily targeted to the CA1 pyramidal cells of the hippocampus. The technique for generating these knock-out mice was detailed previously (Tsien et al., 1996a). NR1 knock-out mice ( $n=36$ in total) were male heterozygous for the viral Cre recombinase gene and homozygous for the floxed NR1 gene. The control group ( $n=52$ in total) included male littermates of three genotypes: homozygous for the floxed NR1 gene, heterozygous for Cre, and wild type. Mice were tested at the age of 1.5-3 months and were housed in standard conditions: $12 \mathrm{~h}$ light/dark, with water and food ad libitum. All experiments were performed in compliance with the European Animal Ethics Committee.

\section{Behavioral testing}

All behavioral analyses were performed blind to the genotype of the mice. All animals were tested according to the S.H.I.R.P.A. protocol (for review, see Crawley, 2000). Animals underwent the tasks presented below in the following order: general appearance, spontaneous behavior and neurological reflexes, the anxiety task, and the "starmaze" task. A different group of mice had already been tested for normal spontaneous activity (Rondi-Reig et al., 2001).

\section{General appearance, spontaneous behavior, and} neurological reflexes

Animals were tested for gross abnormalities that could interfere with behavioral testing. General appearance was evaluated by observing the presence of whiskers, the absence of palpebral closure, and piloerection. Body weight was recorded. Each mouse was then placed for $3 \mathrm{~min}$ in an unfamiliar standard mouse cage for observation of any abnormal spontaneous behavior. Examples of aberrant actions included wild running, constant circling, abnormal jumping, licking, and frozen immobility. Three neurological reflexes were studied according to established protocols (Paylor et al., 1998) (for review, see Crawley, 2000): eyeblink reflex, ear twitch reflex, and whisker-orienting reflex. The eyeblink reflex was tested by approaching the eye with the tip of a clean cotton swab. Normal mice will close their eye. The ear twitch reflex was tested by touching the ear with the tip of a clean cotton swab. Normal mice will flip their ear back to their head. The whisker-orienting reflex was tested by lightly brushing the whiskers of a freely moving animal with a small paint brush. Normal mice will stop moving their whiskers when they are touched, and they may turn the head to the side on which the whiskers were touched. Because these are reflexes, the different responses cannot be quantified in a graded way. We determined whether animals either presented a re-
Multiple strategies version of the starmaze

Probe test

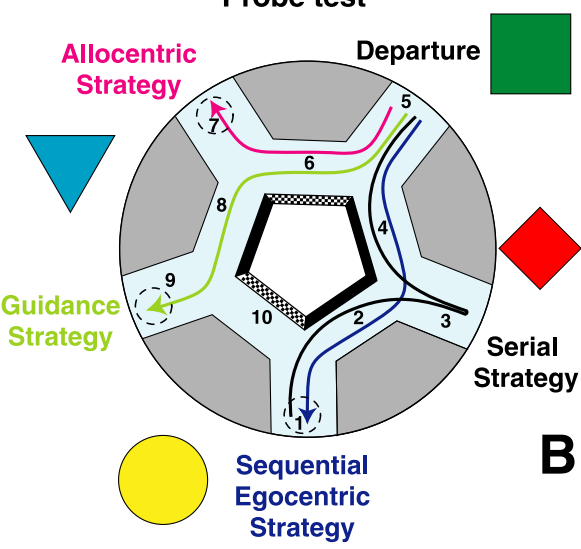

Sequentia

Strategy

Figure 1. The starmaze task: the multiple strategies version. $\boldsymbol{A}$, The training part. The apparatus has five central alleys forming (he probe test. $B$, Characterization of the changed and the animal was placed in alley 5. The position of the distal cues remained constant during the entire training session (i.e. (5-6-8 a serial strategy). Other behaviors were considered as no clear strategy.

sponse to the stimulus or they did not. All mice included in the study presented normal neurological reflexes.

\section{The anxiety task}

The classical elevated plus maze (Pellow et al., 1985) was used for this test. It is a cross-shaped maze with two high-walled arms and two wall-less arms. Each arm was $24 \mathrm{~cm}$ long and $8 \mathrm{~cm}$ wide. Walls were $20 \mathrm{~cm}$ high. The whole apparatus was elevated $1 \mathrm{~m}$ above the floor. Previous studies have shown that anxious mice spend more time in the walled arms than in the open ones. Anxiolytic treatment significantly increases the number of entries in open arms, whereas anxiogenic treatment reduces it (Lister, 1987). We measured the percentage of number of entries and of time spent by the animals in the open arms. An entry was considered effective when the animal placed its four paws in the arm. The test lasted for $5 \mathrm{~min}$.

\section{The navigation task: the starmaze}

To identify the navigation strategy spontaneously used by an animal, we designed a new task (the starmaze) allowing us to characterize "multiple strategies": allocentric, guidance, egocentric (both simple and sequentially organized), and serial. The starmaze consists of five alleys forming a central pentagonal ring and five alleys radiating from the vertices of this pentagonal ring (Fig. $1 \mathrm{~A}$ ). Each radial alley is $47 \mathrm{~cm}$ long and $25 \mathrm{~cm}$ wide. The entire maze is inscribed in a circle (diameter, $204 \mathrm{~cm}$ ), and all of the alleys are filled with water made opaque with an inert nontoxic product (Acusol OP301; Brenntage, Lyon, France). To solve the task, animals have to swim to a platform hidden below the water surface (Fig. 1A). Intramaze cues in the form of distinct wall coverings (either chessboardlike or black or white) are stick to the inner walls of the central pentagonal ring in a specific order (Fig. $1 A, B$ ). The walls of the radiating alleys are all white. The maze was placed at a fixed location inside a large room (50 $\mathrm{m}^{2}$ ) with fixed three-dimensional extramaze cues. Data were recorded via a video data acquisition system (Martin et al., 1999).

Three paradigms of the task were used. (1) The multiple strategies version, permitting the identification of the learning strategy spontaneously used by an animal when multiple strategies were available. We 


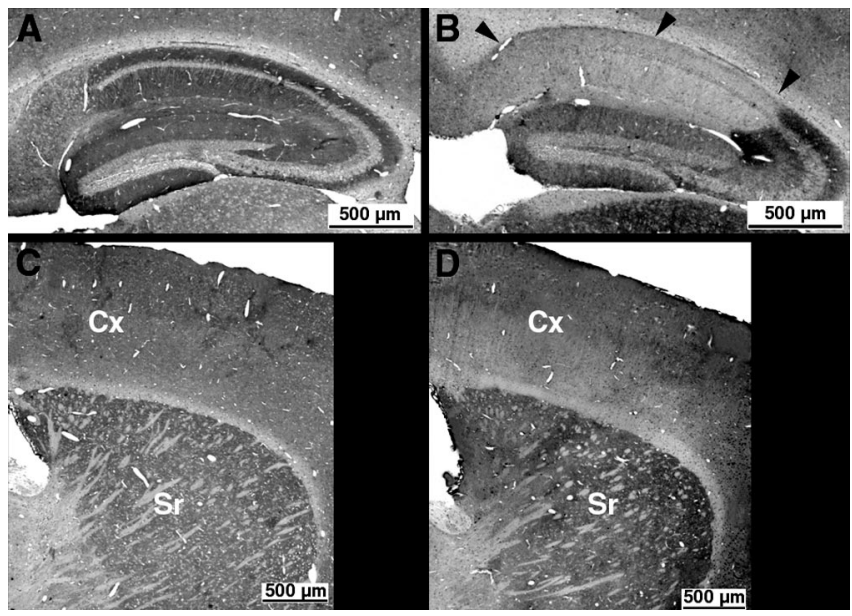

Figure 2. $A-D$, Immunoperoxidase with NR1 antibody performed on control $(A, C)$ and mutant $(\boldsymbol{B}, \boldsymbol{D})$ 3-month- and 2-week-old mice. Compare the hippocampus $(\boldsymbol{A}-\boldsymbol{B})$, the striatum, and the cortical areas $(\boldsymbol{C}-\boldsymbol{D})$ of control and mutant mice. Notice the complete absence of immunoreactivity in the CA1 region of the hippocampus of mutant mice (arrowheads), compared with the partial decrease in the cortex (Cx) (restricted to the deep cortical layer). No decrease is observed in the other area of the brain, in particular in the striatum (Sr).

tested a first group of animals including $n=32$ controls and $n=15$ mutants. (2) The egocentric version, rewarding the same sequence of movements from different starting points. Two protocols were used, a "one-body turn" and a "two-body turn." A second group of naive mice, including $n=9$ controls and $n=9$ mutants, was tested successively in these two protocols. (3) The allocentric version of the task, testing the ability to encode spatial information allocentrically to locate the fixed platform from randomly chosen departure points. In this allocentric version of the task, we tested some of the animals that underwent the multiple strategies paradigm. This third group included $n=11$ controls and $n=12$ mutants.

The multiple strategies version of the starmaze task. This was designed to permit the identification of a learning strategy spontaneously used by an animal. To achieve this characterization, we imagined a task with two complementary components: the training part and the probe test. The training part was composed of two trials per day during $20 \mathrm{~d}$. One probe test was inserted between these two training trials every $5 \mathrm{~d}$. The probe test was performed between the two training trials of the day to reduce the possibility of interference between the probe test and the training part.

Therefore, on day 5, 10, 15, and 20, animals underwent three trials (two training trials and one probe test). Because the entire session lasted $20 \mathrm{~d}$, four probe tests were performed in total. A control test was performed after the $20 \mathrm{~d}$ of training to confirm the interpretation of the results of the probe tests. Both, extramaze and intramaze cues were removed and a circular black curtain was placed around the maze (cue removal condition). In this situation, animals could only refer to idiothetic cues and therefore rely on an egocentric strategy. We quantified the number of errors made by each mouse by comparing their trajectory to an ideal egocentric path (turning left-right-left).

During a training trial, an animal was placed in the maze alley number 1 and it had to find the escape platform located in alley 7 (Fig. $1 \mathrm{~A}$ ). During a probe test trial, the animal had to find the platform starting from alley 5. The measured parameters were the mean escape latency (i.e., time to reach the platform), the number of visited alleys and the trajectory used to find the platform. During the training part, we also quantified the percentage of left turns performed at the three encountered intersections (I, II, III) (see Fig. 6A). If an animal was unable to find the platform within $90 \mathrm{~s}$, it was taken by hand to the platform. In all cases, the animal was left on the platform for $30 \mathrm{~s}$.

When an animal was trained from a fixed departure point (alley 1) to find a fixed goal located in alley 7 (training part), it could use different sensory information to learn and then perform the optimal trajectory to the goal (Fig. $1 A$, black arrow). Depending on the sensory information preferentially used by an animal, four different strategies were defined. An animal spontaneously using the configuration of distal visual cues was considered to employ an allocentric strategy. The ability to follow a sequence of intramaze cues was called guidance. The use of a sequence of self-movements (e.g., turning left-right-left) was called sequentialegocentric strategy. This latter strategy can be understood in terms of associations of stimulus-response-stimulus behaviors, which requires the execution of a specific sequence of body rotations associated to an ensemble of choice points (sequence learning). Finally, we called serial strategy the solution consisting of visiting all radiating alleys successively and systematically to reach the goal. Notice that the serial strategy does not require any learning of the sequential organization of the body movements. Rather, an animal using this strategy just explores all encountered alleys by turning toward a defined direction independently of specific choice points.

The departure point of the probe test (i.e., alley 5) was selected to identify which of the four possible strategies was spontaneously used by the animal. Indeed, alley 5 is the only point from which the different navigation strategies can be dissociated according to the trajectory of the animal (Fig. $1 \mathrm{~B}$ ). The probe test relies on the assumption that an animal occasionally placed at a different departure point will continue to use the same cues it used during training. This working hypothesis has been already successfully adopted in the T-maze protocol (Packard and McGaugh, 1996). Therefore, if the animal preferentially used the distal visual cues during training (departure point alley 1), it will continue to use them when placed at the departure location 5 , and it will reach the goal located in alley 7 . In contrast, if during training, the animal was guided by the proximal visual cues, then during the probe test, he will first swim toward the chessboard-like wall, then toward the black wall, and finally toward the white one and therefore arriving at alley 9. If during training the animal learned to turn left-right-left, then during the probe test, it will arrive at the alley 1 . Finally, if it merely learned to first turn to the left and then explore all the alleys successively (serial navigation), it will also arrive at the alley 1 but it will first visit the alley 3. During the probe test, three platforms were placed at locations 7,9 , and 1 .

We quantified the percentage of animals using each of these four strategies. The important parameter taken into account was the trajectory used to swim to the goal alley. Therefore, after the above explanations, the allocentric strategy would correspond to the 5-6-7 trajectory, the sequential egocentric to the $5-4-2-1$ trajectory, the guidance to the $5-6-8-9$ path, and the serial strategy to the $5-4-3-2-1$ path. During the probe test, the animal was considered as having visited an alley if it had entered it at least half-way. To avoid an eventual learning of the two new platform positions ( 7 and 9 ), during the probe test the animal was removed from the platform as soon as he had climbed on it.

The egocentric version of the starmaze task. This included two protocols: a "one body turn" and a "two body turns." In both protocols, all the intramaze cues were removed, and the extramaze cues were masked with a black circular curtain around the maze, therefore forcing the animal to use an egocentric strategy. Everyday, the animal was placed at four random starting locations. In the "one body turn" protocol, the maze was reduced to a Y maze by blocking the end of the alleys after the first intersection. The animal had to learn to turn left to find the platform (see Fig. $7 A$ ). The duration of the training period was $13 \mathrm{~d}$, four trials a day. In the "two body turns" protocol, animals had to learn to turn to the left and then to the right to reach the goal. At all intersections, the end of the right alley was blocked (see Fig. $7 B$ ). Animals were trained for $15 \mathrm{~d}$, and we measured the percentage of left turns at each encountered intersection. The animals were removed from the platform as soon as they had climbed on it.

The allocentric version of the starmaze. This lasted $9 \mathrm{~d}$. The platform was always located in alley 7 . The intramaze cues were removed and the mouse was placed at a randomly selected alley not containing the platform (i.e., alleys 1, 3, 5, and 9) (see Fig. 8A). Each mouse was given four trials per day, each trial corresponding to a randomly selected departure alley. If an animal was not able to find the platform by itself within $90 \mathrm{~s}$, it was taken by hand to the platform. In either case, the animal was left for 30 s on the platform. Similar to the "multiple strategies" learning version, 
the measured parameters were the mean escape latency, the sequence, and the number of visited alleys to find the platform. In addition, we measured the percentage of left turns at the first intersection. We also quantified the errors performed by the mice by comparing their trajectory to the ideal path toward the goal.

\section{Histological verification}

After the behavioral experiments, mice were anesthetized with sodium pentobarbital (100 $\mathrm{mg} / \mathrm{kg}$ body weight, i.p.) and perfused through the heart with $40 \mathrm{ml}$ of $4 \%$ paraformaldehyde in $0.1 \mathrm{~m}$ sodium phosphate buffer (PBS), $\mathrm{pH}$ 7.4. Brains were removed immediately after perfusion and immersed in fresh fixative (paraformaldehyde, $0.1 \mathrm{M}$ ) overnight at room temperature (RT). Brains were then embedded in paraffin after dehydration using a graded alcohol series. Sagittal sections $(5 \mu \mathrm{m})$ were cut and mounted on glass slides (Superfrost/Plus; Bioblock Scientific, Illirch, France). Sections were pretreated with pepsin $(1 \mathrm{mg} / \mathrm{ml}$; Dako France, Trappes, France) in $0.2 \mathrm{M} \mathrm{HCl}$ at $37^{\circ} \mathrm{C}$ for $10 \mathrm{~min}$, as reported by Fukaya et al. (2003). After blocking with $10 \%$ normal goat serum in PBS, sections were incubated in primary antibody (rabbit polyclonal antibody against the rat NR1 subunit (AB 1516; Chemicon, Euromedex France, Mundolsheim, France) diluted to a final concentration of $3 \mu \mathrm{g} / \mathrm{ml}$, at $4^{\circ} \mathrm{C}$ for $2 \mathrm{~d}$. The sections were then incubated with biotinylated goat anti-rabbit IgG (Vector Laboratories, Abcys, Paris, France) at RT for $1 \mathrm{~h}$ and avidin-biotin peroxidase (Elite standard; Vector Laboratories) complex at RT for 30 min. Immunoreaction was visualized with diaminobenzidine.

\section{Results}

A lack of NMDARs in the CA1 area of the hippocampus and a decrease of these NMDARs in the deep cortical layers

The distribution of the NR1 gene knockout was determined between 2.5- and 3.5month-old mice, after behavioral experiments. The results showed that the NR1 knockout in the hippocampus was restricted to the CA1 area, and did not expand to the CA3 or the dentate gyrus even at 3 months and 2 weeks of age (Fig. $2 A, B)$. A reduction of NR1 immunoreactivity was observed in the cortex, restricted to the deep cortical layer. No reduction in immunoreactivity was observed in other areas of the brain including the striatum of the mutant mice (Fig. 2C,D). These results are in agreement with those reported by Fukaya et al. (2003).

\section{NR1-KO and control mice appeared healthy and well groomed}

No abnormalities were observed in general appearance, spontaneous behavior and neurological reflexes (S.H.I.R.P.A.). Anxiety, as measured with the elevated plus maze (Lister, 1987), revealed no significant difference between mutant and control mice in

\section{Multiple strategies version of the starmaze: the training part}
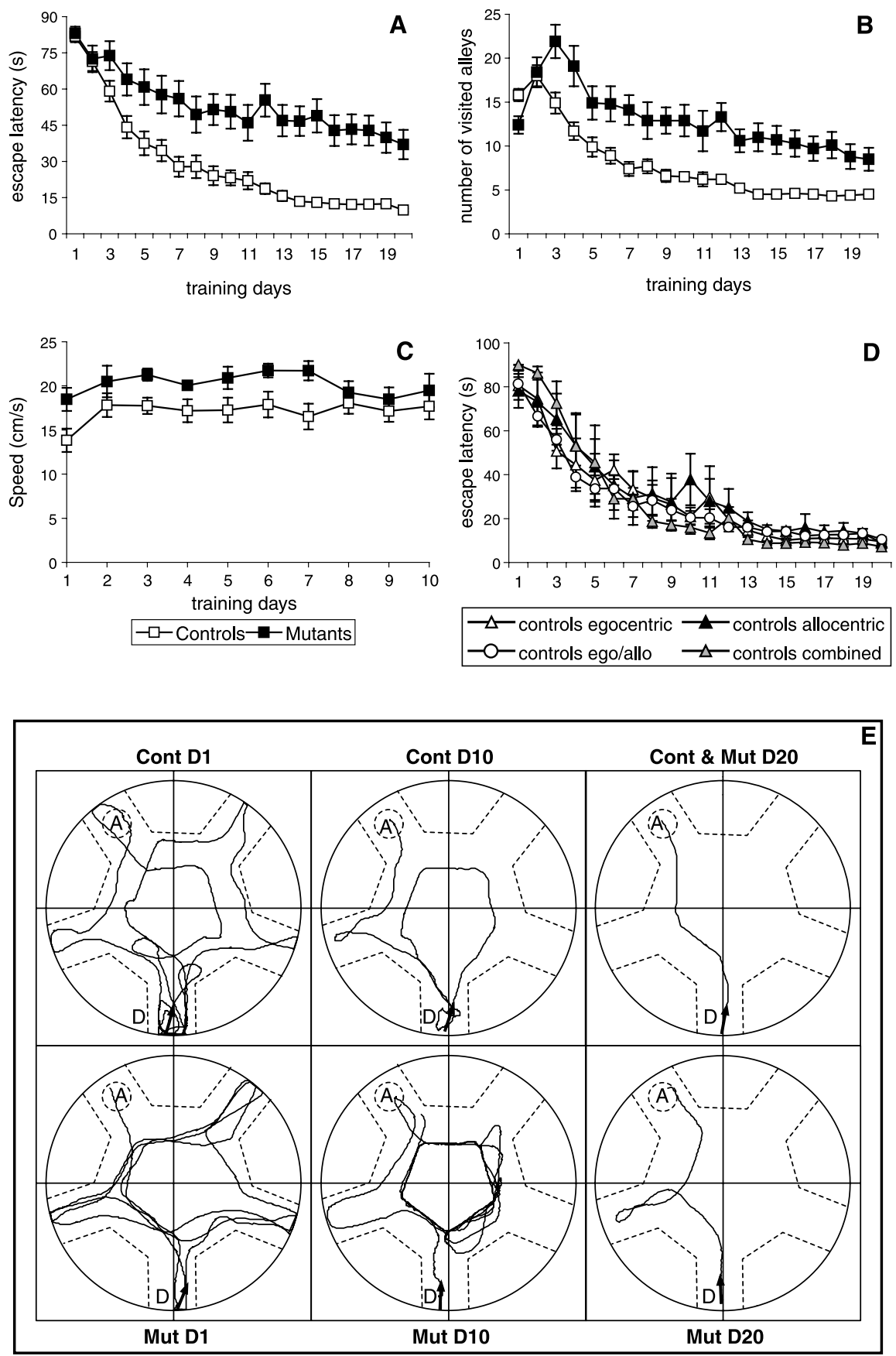

Figure 3. The multiple strategies version of the starmaze: the training part. The escape latency, the speed, and the number of alleys visited is represented for controls $(n=32)$ and mutants $(n=15)$. $\boldsymbol{A}$, The time (in seconds) to reach the hidden escape platform. $\boldsymbol{B}$, The number of alleys visited to reach the platform over time. $\boldsymbol{C}$, The swimming speed (in centimeters per second). $\boldsymbol{D}$, The escape latency (in seconds) of the four groups of control mice using either allocentric, or egocentric, or both strategies (egocentric/egocentric learners), or a combination of either serial/guidance with allocentric/egocentric strategies (combined learners) during the training period. Notice that the strategy used had no effect on the performance. $\boldsymbol{E}$, Representative swimming trajectories of the animals. Three sample trajectories are shown for controls (ContD1, ContD10, and Cont \& MutD20) and four sample trajectories are displayed for NR1-K0 mice (MutD1, MutD10, MutD20, and Cont \& MutD20). These examples correspond to the day 1 (ContD1, MutD1), day 10 (ContD10, MutD10) and day 20 (Cont \& MutD20, MutD20) of training. neither the percentage of time spent in the open arms (control, $25 \pm 6$; mutants, $37 \pm 7$; ANOVA, $\left.F_{(1,27)}=2.35, p=0.13\right)$, nor the percentage of open arm entries (control, $29 \pm 5$; mutants, $38 \pm 6$; $\left.\operatorname{ANOVA}, F_{(1,27)}=1.87, p=0.18\right)$. 
Multiple strategies version of the starmaze: the probe test
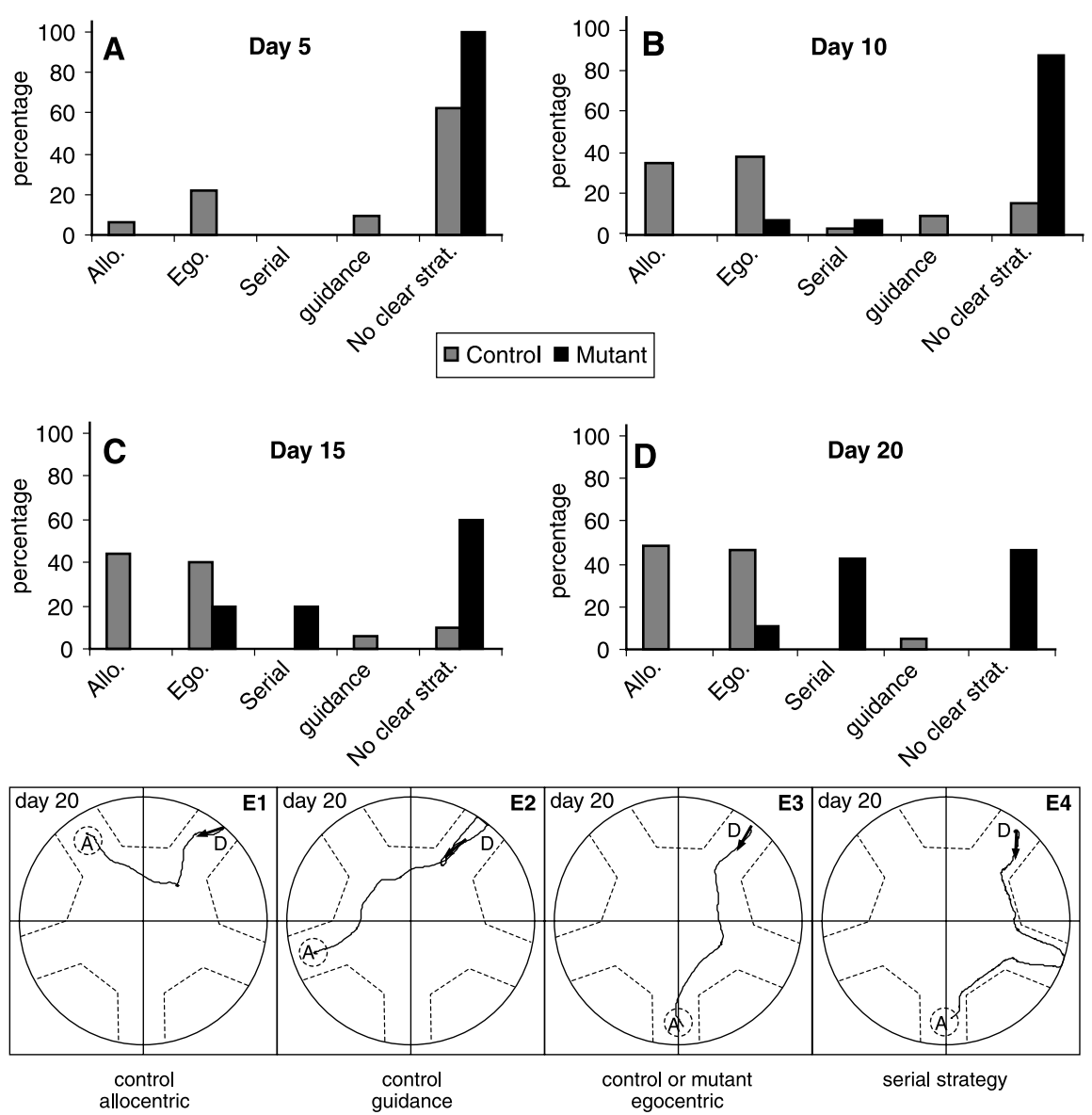

Figure 4. The multiple strategies version of the starmaze: the probe test. We characterized the strategies used to solve the starmaze task. The strategy used by each animal is revealed by the probe test. $\boldsymbol{A}-\boldsymbol{D}$, The percentage of animals using a given strategy is represented on day $5(\boldsymbol{A})$, day $10(\boldsymbol{B})$, day $15(\boldsymbol{C})$, and day $20(\boldsymbol{D})$. $\boldsymbol{E}$, Examples of trajectories for mice using an allocentric $(\boldsymbol{E 1})$, a guidance $(\boldsymbol{E} 2)$, a sequential-egocentric $(\boldsymbol{E 3})$, or a serial (E4) strategy.

\section{A coexistence of multiple strategies of navigation revealed by the starmaze}

The analysis of the training part of the multiple strategies version of the task showed that control mice rapidly learned the task (Fig. 3). After $14 \mathrm{~d}$ of training, the time to reach the platform (mean escape latency) (ANOVA, $F_{(1,19)}=39.05, p<0.0001$ ) and the number of visited alleys (ANOVA, $F_{(1,19)}=28.6, p<0.0001$ ) decreased significantly to a plateau value of $15 \pm 2 \mathrm{~s}$ and $5 \pm 1$ visited alleys, respectively (Fig. $3 A, B$ ).

The four probe tests performed on days 5, 10, 15, and 20 demonstrated that control mice essentially used egocentric and allocentric strategies. The guidance and serial strategies were seldom used (Fig. 4). Examples of these different strategies are presented in Figure 4E1-E4.

The majority of the control animals (60\%) developed the ability to switch back and forth between strategies, meaning that they used different strategies during the four probe tests. This suggests coexistence in the acquisition of the different strategies. The other $40 \%$ of control animals used exclusively either the allocentric (19\%) or the sequential-egocentric $(21 \%)$ strategy during the four probe tests. None of the control animals used guidance or serial strategies exclusively. Guidance and serial strategies were systematically observed in combination with egocentric and/or allocentric strategies (data not shown).
Therefore, four groups of mice were characterized. Those using the egocentric (allocentric) strategy during the four consecutive probe tests were labeled as egocentric (allocentric) learners. Those combining both strategies were named allocentric/egocentric learners (35\%). The fourth category was composed of animals switching between guidance/serial strategies and egocentric/allocentric strategies (combined learners, 25\%).

The use of different strategies did not necessarily correspond to better or worse performance. The learning curve of control mice using the different possible strategies was analyzed (Fig. 3D). No difference was observed between the four groups of control mice, demonstrating that the different strategies led to equivalent performances (ANOVA, $F_{(3,28)}=0.27, p=$ $0.85)$.

The interpretation of the strategy used by the mice during the probe test was confirmed by the control experiments executed after the training session of $20 \mathrm{~d}$ of the multiple strategies version of the task. These control tests used either a cue removal condition (Fig. $5 A, C$ ) or the random departure condition (allocentric version) (Fig. $5 B, D)$.

The results clearly demonstrated that animals classified as allocentric learners made several errors in the cue removal condition $(5.8 \pm 1.1)$ (Fig. $5 A)$. Their trajectories indicated that they were either lost (Fig. 5C1) or that they relied on a serial strategy (Fig. 5C2). In contrast, in the random departure condition, allocentric learners were able to solve the task with a minimum of errors $(1.0 \pm 0.5)$ (Fig. $5 B, D 1, D 2)$.

Conversely, and as expected, mice classified as egocentric learners were perfectly able to find their way despite the cue removal. They reproduced the left-right-left sequence they previously learned (Fig. 5C3,C4). No error was indeed observed when the egocentric learners were tested in the cue removal condition (Fig. 5A). In the random departure condition, they tended to solve the task by performing an egocentric path (left-right-left). As a consequence, they did not find the platform and therefore they finally resorted to a serial strategy (Fig. 5D3,D4). This led to an increased number of errors (3.6 \pm 0.8 ) (Fig. $5 B$ ). These results are in exact correspondence with what we could expect from the classification of the mice as allocentric or egocentric learners using the probe test.

In addition, combined allocentric/egocentric learners were able to solve the two control tests with a minimum number of errors ( $2.5 \pm 0.8$ and $1.4 \pm 0.5$, respectively) (Fig. $5 A, B)$. In the cue removal condition, the majority of them found the goal with very few hesitations, which could reflect the research of visual cues that were removed in this case (Fig. 5C5,C6). In the random departure condition, two kinds of behaviors were observed. Part of the mice went directly to the goal according to their allocentric coding (Fig. 5D6). The others first relied on their egocentric strategy beginning to execute the left-right-left sequence, but they 
finally corrected their trajectory to arrive at the allocentric goal (Fig. 5D5).

Finally, mice classified as combined learners (including all other combinations between either guidance/serial strategies and allocentric/egocentric paths) showed an intermediate score between allocentric and egocentric learners $(4.1 \pm 2.1$ and $1.8 \pm 0.8$, respectively) (Fig. $5 A, B$ ). Mice were often lost (Fig. 5C7,D8). This is for example the case in the cue removal condition for a learner combining an allocentric with a guidance strategy (Fig. 5C7). Likewise, an intermediate score was obtained in the random departure condition by learners combining guidance and egocentric strategies (Fig. 5D8). Other combined learners were relying on the strategy already used in the probe test and corresponding to the egocentric strategy (Fig. $5 C 8$ ) or the allocentric strategy (Fig. 5D7).

In addition, our results suggest that the serial strategy is likely to be used when an animal is lost. Indeed, in the cue removal test, mice classified as allocentric learners often resorted to a serial strategy, although they had not been using it previously (Fig. $5 C 2)$. Similarly, mice labeled as egocentric learners often resorted to the serial strategy when tested in the random departure version of the task (Fig. 5D3,D4).

\section{The multiple strategies version of the} starmaze task demonstrates that NR1$\mathrm{KO}$ mice are impaired in acquiring the memory of the platform location

NR1-KO mice showed performance impairments when compared with controls in terms of both escape latency (ANOVA, $\left.F_{(1,45)}=47.56, p<0.0001\right)$ and number of visited alleys (ANOVA, $F_{(1,45)}=42.17, p<$ 0.0001 ) (Fig. $3 A, B$ ). There was a significant interaction between the trial and the genotype in both cases (ANOVA, $F_{(1,19)}=$ 37.61 or $36.66, p<0.0001)$. The increased escape latency of NR1-KO mice was not attributable to a reduced swimming speed, as NR1-KO mice actually swam slightly faster than control mice (Fig. $3 C$ ). The behavior of NR1-KO mice appeared less efficient compared with control mice. Although both groups of mice explored the entire maze on the first training day (Fig. 3, compare ContD1 and MutD1), a clear difference appeared on day 10 . In contrast to NR1-KO mice, control mice visited fewer alleys (Fig. 3, compare ContD10 and MutD10). At the end of the training period (day 20), all control mice had learned the task and swam directly to the goal alley (Fig. 3, Cont and MutD20). Only $47 \%$ of NR1-KO mice were able to swim to the goal alley either directly (1-10-8-7) (Fig. 3, Cont and MutD20) or indirectly (1-10-9-8-7) (Fig. 3E, MutD20).

C Cue removal hoc analysis; $p<0.05)$.

\section{Control tests}
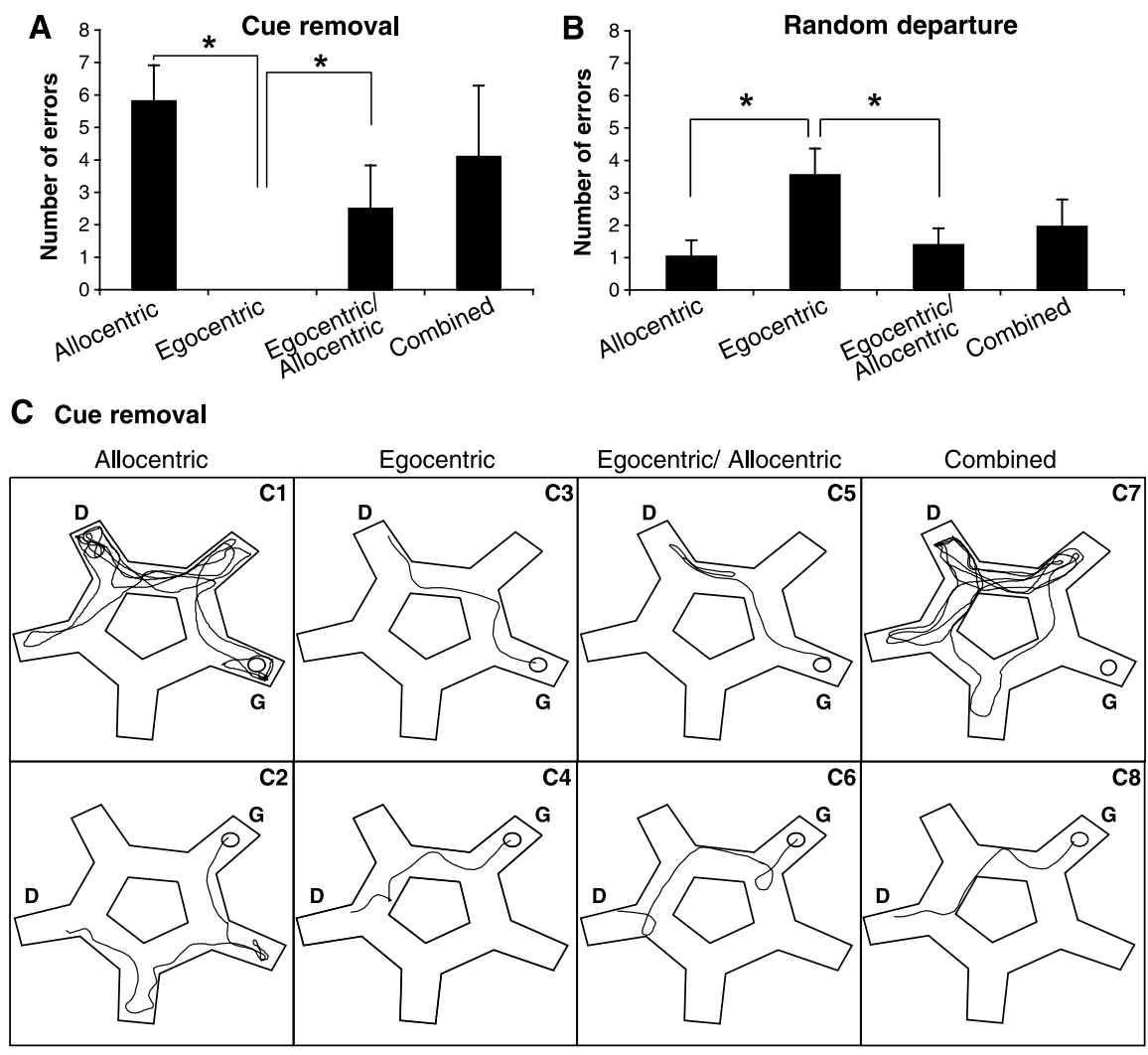

D Random departure

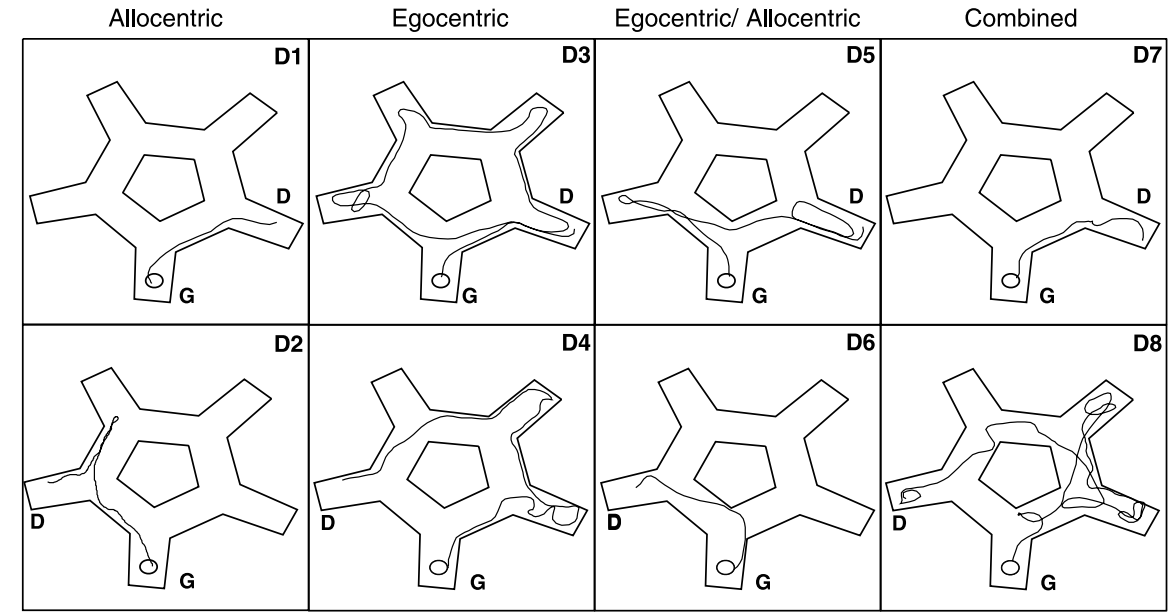

Figure 5. The control tests: cue removal and random departure control tests. $\boldsymbol{A}$, The cue removal situation. Extramaze and intramaze cues were removed and a circular black curtain was placed around the maze. In such a situation, animals can only refer to idiothetic cues. We quantified the number of errors made by each mouse by comparison to an ideal egocentric path (turning left-right-left) depending on the previously demonstrated strategies. $\boldsymbol{B}$, Random departure situation. We forced animals to use an allocentric strategy by removing proximal intramaze cues and placing each animal at a randomly selected departure alley although the goal alley remained the same. In this paradigm, we quantified the errors performed by the mice compared with the shortest trajectory toward the goal. C, D, Examples of trajectory performed by the four groups of mice classified as allocentric, egocentric, egocentric/allocentric, or combined learners during the probe tests. $\boldsymbol{D}$ corresponds to the departure point and $\mathbf{G}$ corresponds to the goal platform. The asterisk indicates a significant difference between the indicated groups (ANOVA and post

We then used the probe test to investigate whether NR1-KO mice used the same strategies as controls to reach the platform during the training part. In striking contrast to control mice, NR1-KO never used the allocentric and guidance strategies (Fig. 


\section{Multiple strategies version of the starmaze}
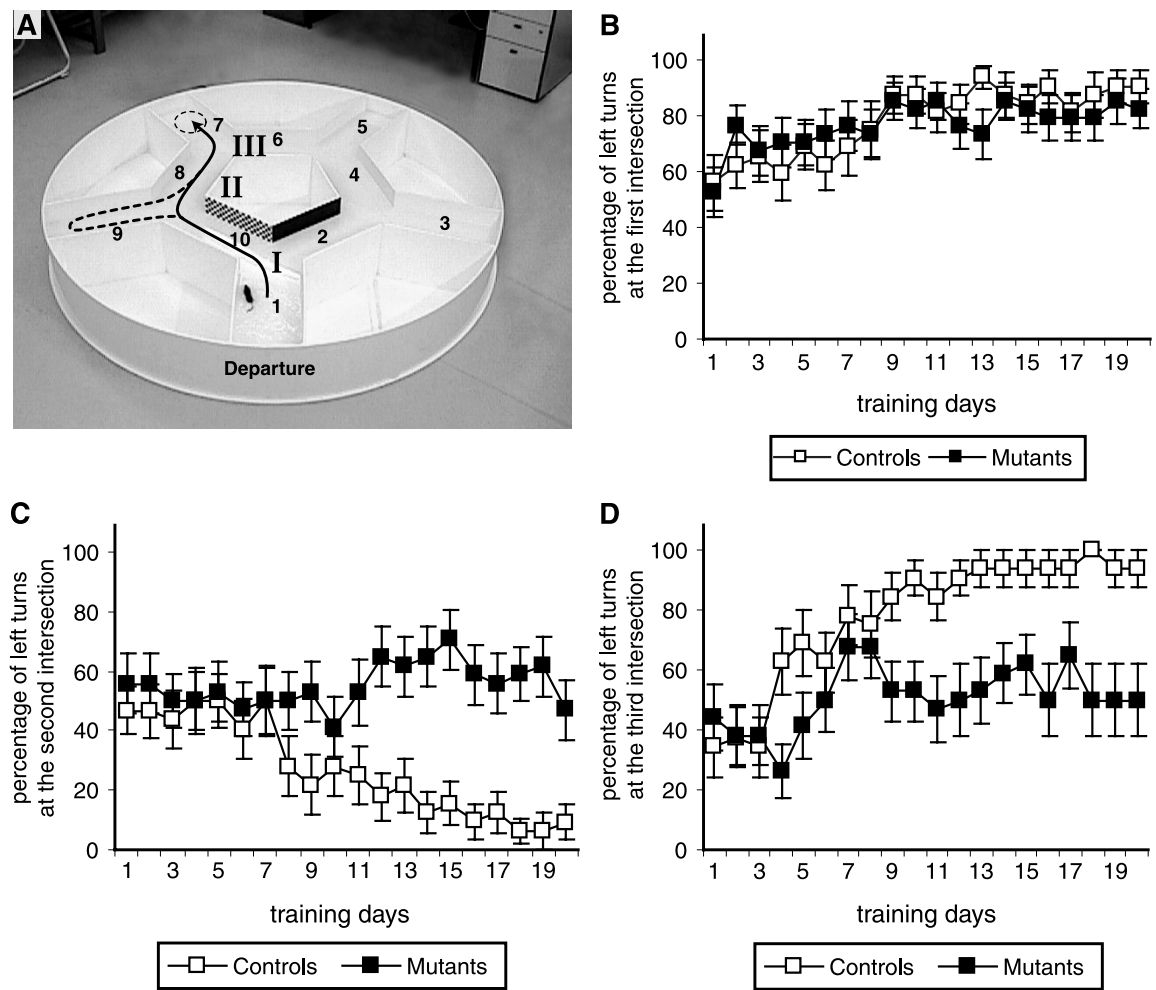

Figure 6. Detailed analysis of the behavior of control and NR1-K0 mice in the multiple strategies learning task. $\boldsymbol{A}$, Each animal had to learn to turn left at the first intersection (I), right at the second (II), and left at the third (III). The percentage of left turns performed by either mutant or control mice are calculated for each of the three intersections leading to the goal (I, II, III). B-D, The percentage of left turns at the first (I), the second (II), and the third intersections (III), respectively. A learned behavior led to an increase of left turns at the first and the third intersections and a decrease at the second one. Chance level corresponds to $50 \%$ of left turns. Notice that mutant mice remained at chance level at the second and the third intersections. intersections (compare scores of mutant and control mice in Fig. $6 B-D)$. These results show that NR1-KO mice were able to learn the first body turn, but they were impaired in acquiring the memory of three successive body turns (left-right-left). This specific deficit demonstrates that the apparent use of the egocentric strategy by NR1-KO mice (Fig. 4) was sometimes observed simply because the NR1-KO mice learned to turn left at the first turn, whereas they had a 50\% chance at the second and third turns.

To demonstrate that the mutants were not simply prevented from adopting an egocentric strategy but they were rather unable to use it, we forced NR1-KO mice to learn a version of the task that rewards the same sequence of movements from different starting points (the egocentric version of the starmaze) (Fig. $7 A, B$ ). In the one body turn protocol, control and mutant mice learned the first intersection successfully within $10 \mathrm{~d}$ of training (ANOVA, $\left.F_{(1,12)}=6.26, p<0.0001\right)$. No significant difference was observed between the two groups (ANOVA, $F_{(1,16)}=1.57, p=0.23$ ) (Fig. 7C). In contrast, in the two body turns protocol (Fig. 7D), NR1-KO mice were clearly impaired. Control mice learned the task after $10 \mathrm{~d}$ of training and consistently turned right (correct choice) at the second intersection. The performance of NR1-KO mice remained at chance level at the second intersection $4 A-D)$. Instead, they tended to adopt either an egocentric or a serial strategy (Fig. $4 B-D$; E3,E4, sample trajectories). The statistical study showed a significant difference in the use of the allocentric, the egocentric, and the serial strategies between control and NR1-KO mice (Chi2, $p<0.05$ ). Concerning the guidance strategy, no statistical difference was observed between control and NR1-KO mice (Chi2, $p>0.05$ ), certainly because NR1-KO mice never used it and control mice used it really seldom.

\section{NR1-KO mice are impaired in the sequential-egocentric but not in the simple egocentric strategy}

We next wanted to determine whether NR1-KO mice were impaired in the learning of an egocentric strategy requiring sequentially organized body movements. We first analyzed in detail the learning behavior of NR1-KO mice in the multiple strategies version of the task. To navigate directly toward the platform, mice had to encounter three specific intersections (I, II, and III) (Fig. $6 A)$, turning successively left-right-left. The percentage of left turns was measured when animals swam out of alley number 1 (first intersection, I), of alley number 10 (second intersection, II) and of alley number 8 (third intersection, III). Learning the task required an increase of the percentage of left turns at intersection points I and III, and a decrease of the percentage of left turns at intersection II (Fig. 6A). After $10 \mathrm{~d}$ of training, $>80 \%$ of the control mice learned the task and therefore turned left at the first intersection, right at the second, and left at the third. In contrast, NR1-KO mice learned to turn left at the first intersection, but they always remained at chance level on the second and third similar to the results of the multiple strategies version of the task (see Fig. 6C).

\section{NR1-KO mice are unable to use the allocentric strategy}

To confirm the inability of NR1-KO mice to adopt an allocentric strategy (observed in the probe tests) (Fig. 4), mutant and control animals were trained to find the platform located at a fixed alley (number 7) from other randomly selected alleys in the absence of intramaze cues (Fig. 8A). NR1-KO mice were clearly impaired compared with control mice (ANOVA, $\left.F_{(1,21)}=8.84, p=0.007\right)$. From the beginning of the training period, all control mice had a better escape latency than NR1-KO mice (Fig. $8 \mathrm{~B}$ ). At the end of training, control mice rapidly swam directly to the goal from any of the departure alleys (Fig. 8C1-C3). In contrast, NR1-KO mice did not improve their performances over training; they never reached the performance level of controls and they presented a plateau of $8 \pm 1$ visited alleys (data not shown). They did not acquire the ability to perform a direct trajectory to the platform, and they rather resorted toward a serial strategy (Fig. 8C4).

To better characterize the behavior of both mutant and control mice in the allocentric version of the task, we then analyzed the percentage of left turns at the very first intersection encountered (see Materials and Methods). The results showed that both controls and mutants performed $\sim 50 \%$ from the first day of training (Fig. $8 D$ ) (ANOVA, $\left.F_{(1,21)}=0.48, p=0.49\right)$. We further analyzed the behavior of NR1-KO mice for each of the four departure points independently (Fig. $8 E$ ). Control mice demonstrated the ability to flexibly adapt their first turning behavior 
depending on the departure point, therefore choosing to turn to the direction leading to the shortest path to the platform. In contrast, NR1-KO mice turned to the correct direction when starting from a distal departure point (i.e., alleys number 1 and 3) but not from a proximal departure location (i.e., alleys 9 and 5).

\section{Discussion}

Using a new spatial navigation paradigm, the starmaze, we found that normal mice solved the task using both allocentric and sequential-egocentric strategies. In contrast, NR1-KO mice were unable to acquire the navigation task using either the allocentric or the sequential-egocentric strategy. These results suggest a role of the NMDAR-dependent mechanisms of the forebrain in the acquisition of information spatially or sequentially linked.

The starmaze task revealed a coexistence of multiple strategies

The design of the starmaze task took inspiration from the combination of the Morris water maze (Morris et al., 1982) and the T-maze (Packard and McGaugh, 1996). Similar to the Morris water maze, the complexity of the task requires developing a map-based (allocentric) strategy. As for the T-maze, alternative strategies can be used and identified. Our new task adds the possibility to test the sequential-egocentric strategy in addition to the map-based one. The egocentric strategy defined in the starmaze, refers to route-based strategy (Arleo and Rondi-Reig, 2006) as it requires a sequential organization of the information. This is different from target approaching or stimulus-triggered response as defined in the T-maze. A control animal can use both allocentric and sequential-egocentric strategies, which supports the idea of coexisting strategies in the rodent brain (Fenton et al., 1998). These two navigation behaviors led to equivalent performances (Fig. 3D). In the T-maze paradigm, control rats used the allocentric strategy at the beginning of the training period, and then shifted to an egocentric one (Packard and McGaugh, 1996). In contrast, control animals trained in the multiple strategies version of the starmaze task continuously alternated between allocentric and egocentric strategies even after $20 \mathrm{~d}$ of training (Fig. $4 D)$. The complexity of the starmaze, compared with the T-maze, might account for this difference and make this task dependent, at least partially, on the hippocampal system. In humans, for example, it has been emphasized that the complexity of the performed task is the primary criterion for the involvement of the hippocampus (Burgess et al., 2002).

\section{Strategies requiring spatial or sequential organization of} information are impaired in NR1-KO mice

In contrast to control mice, NR1-KO mice were unable to use the allocentric strategy in the multiple strategies (Fig. 4) and in the allocentric versions of the starmaze (Fig. 8). Detailed analysis of the behavior of these mutants during the multiple strategies and the egocentric version of the starmaze task also revealed a deficit

\section{Egocentric version of the starmaze}
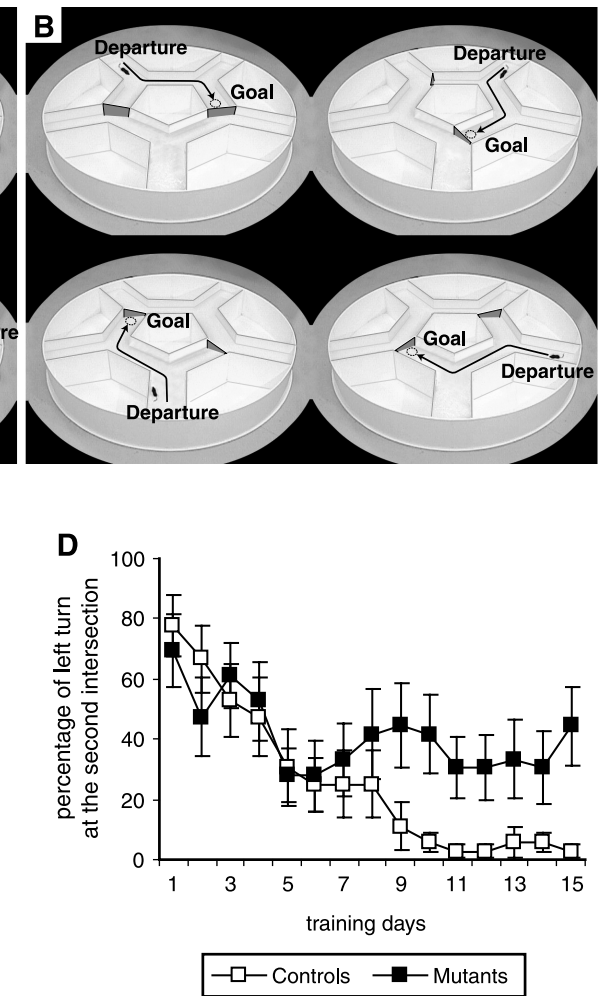

- Mutants

Figure 7. The egocentric version of the starmaze. We compared mutant $(n=9)$ and control $(n=9)$ mice. $\boldsymbol{A}$, The one body turn . The maze was reduced to a Y maze. $\boldsymbol{B}$, The two body turn protocol. The animals had to turn left and then right to reach starting locations. $C, D$, The percentage of left turns at the first and the second intersections, respectively. Notice that mutant mice remained at chance level at the second intersection.

in the ability to acquire the memory of successive body turns (Figs. 6, 7). In contrast to control mice, which correctly learned the left-right-left turning sequence, NR1-KO mice learned to turn left at the first intersection but they remained at chance level at the second (Figs. 6, 7) and third intersection points (Fig. 6), showing a deficit in the sequential-egocentric strategy.

These deficits can be discussed according to theoretical perspectives proposing a role of the hippocampus in either spatial memory (O'Keefe and Nadel, 1978), or behavioral inhibition, [i.e., the ability to inhibit inappropriate response (Jarrard, 1973; McNaughton, 1997; Chan et al., 2001; Tracy et al., 2001), or relational memory (Eichenbaum et al., 1999)].

The deficit we observed in the allocentric strategy is in agreement with the hypothesis that the NMDARs of the CA1 area of the hippocampus play a crucial role in encoding allocentric knowledge of the environment (Morris et al., 1986; Tsien et al., 1996b; Nakazawa et al., 2004). Place field in the CA1 area of NR1-KO mice are known to be less robust than in controls; this property could explain the deficit in mnemonic associations needed to perform navigation tasks using the allocentric strategy (McHugh et al., 1996).

In the multiple strategies and egocentric version of the starmaze task, NR1-KO mice were able to learn the first body turn but were impaired in acquiring the second and third body turns. This impairment could have been explained in terms of a deficit in inhibiting the previously learned response (first body turn). To address this issue, we took advantage of the group of mice first 

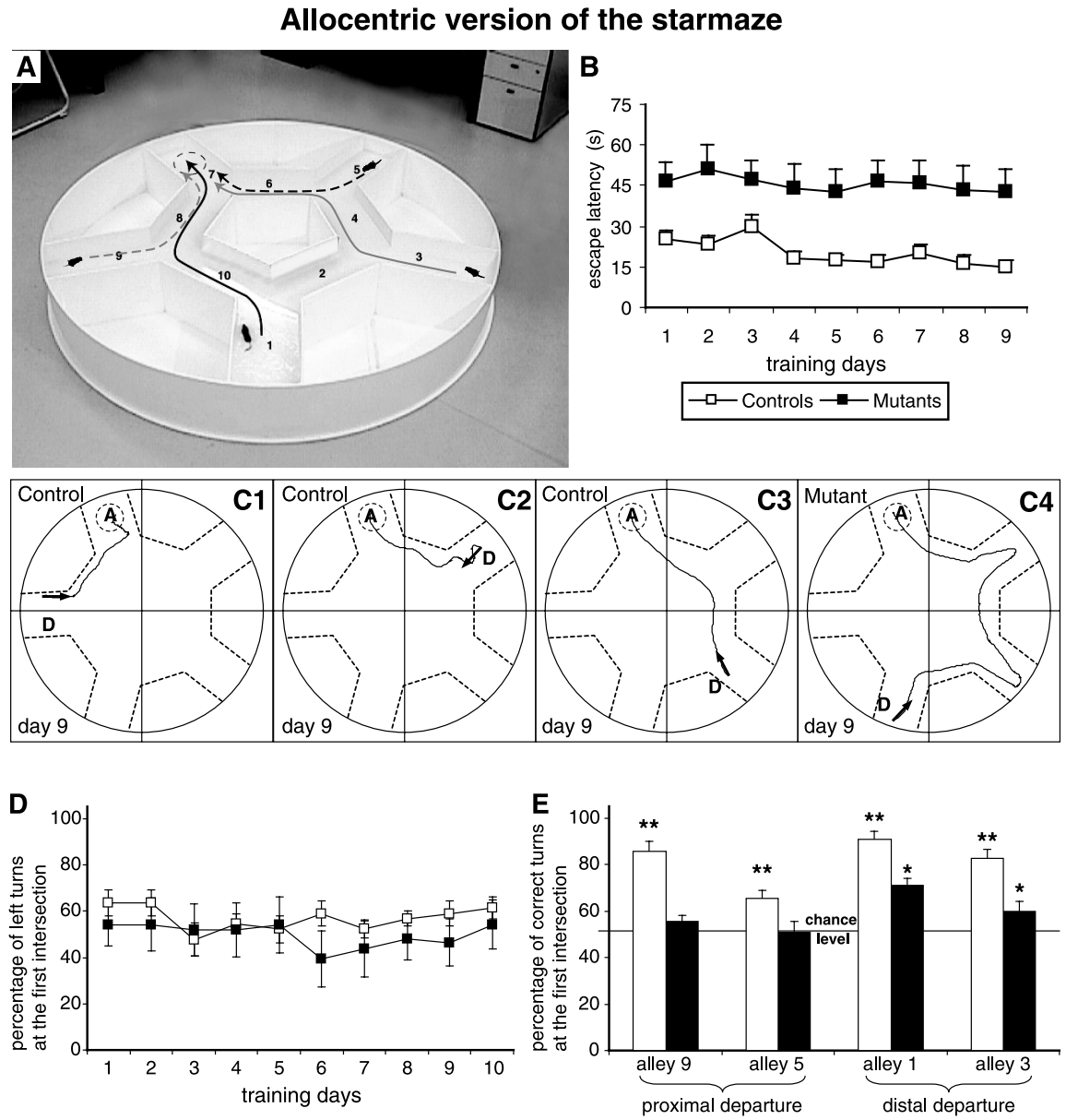

a Controls Mutants

Figure 8. $A$, The allocentric version of the starmaze. The four possible randomly chosen departure locations are represented by the mice. $\boldsymbol{B}$, The time to reach the escape platform (in seconds) is plotted as a function of training days for controls $(n=11)$ and mutants $(n=12)$. Each training day represents the average of the scores obtained during the four trials of a day. C1, C2, C, An example of a control mouse using the shortest trajectory to reach the target alley (represented by a dashed circle) from different random departure points (represented by an arrow). In contrast, C4 is an example of the trajectory of a NR1-K0 mouse, which did resort to a serial strategy to reach the target alley. $\boldsymbol{D}$, The percentage of left turns at the first intersection. For each training day, the percentage corresponds to the mean \pm SEM of the score obtained by all animals of a group at the four departure points. $\boldsymbol{E}$, The percentage of turns toward the platform location (correct turns) at the first intersection encountered depending on the four different departure points. An asterisk denotes a significant difference between NR1-KO mice and chance level. Two asterisks indicate that the score of controls was significantly different from both NR1-K0 mice and chance level (ANOVA and post hoc analysis; $p<0.05)$.

trained in the multiple strategies version of the task and then in the allocentric version. Based on the inhibitory learning hypothesis, NR1-KO mice which learned to turn left at the first intersection in the multiple strategies version, should all have continued to turn left when subsequently trained in the allocentric version. We analyzed the percentage of left turns performed by both control and NR1-KO mice at the first intersection encountered when departing from the four different starting points of the allocentric version of the starmaze (Fig. $8 D$ ). The analysis revealed that both controls and mutants performed $\sim 50 \%$ from the first day of training (Fig. $8 D$ ). These results demonstrate that NR1-KO mice did not present any deficit in the ability to inhibit a previously learned response.

Why were NR1-KO mice able to learn the first body turn but were impaired in acquiring the second (multiple strategies and egocentric versions) and the third body turns (multiple strategies version)? In the egocentric version of the task, the first body turn might have been learned using a simple stimulus-response association as described previously for the T-maze (Packard and McGaugh, 1996). We further analyzed the behavior of NR1-KO mice at each of the four departure points independently in the allocentric strategy (Fig. $8 E$ ). Control mice showed the ability to flexibly adapt their first turning behavior according to the shortest path, suggesting that controls encoded an allocentric representation of the environment. In contrast, mutants turned to the correct direction when starting from a distal departure points but not from a proximal departure (Fig. $8 E$ ). This suggests that NR1-KO mice could learn to turn left or right according to a distal cue located behind the goal alley. This cue could be visible from distal departure alleys but not from proximal ones because of the walls of the starmaze. They seem to have a preserved ability to acquire a simple association. In the allocentric version of the task, mutants learned to associate a visual distal cue with the goal. NR1-KO mice might have used this same simple association to learn the first body turns in the multiple strategies version of the starmaze task (Fig. 6A). Our results suggest that the structures concerned by the lack of NMDA receptors in our model are not involved in the acquisition of a simple association. These are consistent with the proposed existence of an egocentric system, independent of the CA1 hippocampal area, for the acquisition of a simple response (Compton, 2001). There is considerable evidence that the dorsal striatum plays a central role in learning situations that involve the formation of associations between specific stimuli and the specific responses that lead to a reward (Packard and Knowlton, 2002; White and McDonald, 2002). When CA1-NMDAdependent mechanisms are impaired, the ability to develop a simple goal-reward association would be preserved but the sequential organization of movements would be impaired. This supports the proposition of a "goal-orientation" function independent of the hippocampus and involving either the prefrontal cortex (Poucet et al., 2004) and/or the striatum (Wiener, 1993). This interpretation is consistent with preserved NMDARs in the striatum of NR1-KO mice (Fig. 2). It also suggests that some function (possibly independent of NMDARs) is preserved in cortical areas, despite the diminution of number of NMDARs in the deep layers of the neocortex.

\section{Implication for the episodic-like memory}

The deficit we observed in the sequential-egocentric and allocentric strategies is consistent with the possible role of the rodent hippocampus in mediating spatiotemporal associations between the multiple events that constitute the elements of an episodic memory (Morris, 2001; Eichenbaum, 2001). NMDAR-mediated function in area CA1 of the hippocampus is crucial for the acqui- 
sition of relationships between multiple stimuli (Huerta et al., 2000; Rondi-Reig et al., 2001). Recent models and properties of CA1 place cells demonstrating that active hippocampal neurons include proportions of neurons that encode current retrospective and prospective information (Frank et al., 2000; Ferbinteanu and Shapiro, 2003). We propose that the organization of a sequentially organized strategy, but not of a simple egocentric one, requires the CA1 area of the hippocampus to encode a context containing both spatial and temporal information. NMDARdependent mechanisms in the CA1 area of the hippocampus would be necessary for organizing temporally the three independent context-response behaviors required at each intersection (Mehta et al., 2000). If NMDAR-dependent mechanisms of the deep cortical layers are involved, we suggest that this spatiotemporal context would then be transferred to cortical areas in agreement with a proposed role of the neocortex in path selection (Poucet et al., 2004) (see supplemental figure, available at www.jneurosci.org as supplemental material). We have to be cautious concerning the functional importance of a diminution rather than a lack of NMDARs in the deep cortical layers. To confirm the proposed complementary roles of NMDARs in the hippocampus and in the deep cortical layers, additional investigations using other animal models and behavioral analyses will be performed in the future.

\section{Conclusion}

The starmaze task is a new paradigm permitting a detailed analysis of different strategies of navigation. Normal animals undertaking this complex task used both the allocentric and the sequential-egocentric strategies. NR1-KO mice were impaired in learning both strategies. The deficit observed in these mice is specific. Only the sequential organization of multiple body rotations was impaired, whereas learning a single body turn appeared intact. This suggests that different areas are involved in these two processes. Our proposition of a role of CA1-NMDARs in the organization of the sequential-egocentric strategy is in agreement with the properties of CA1 place cells and with theoretical models suggesting a role of the hippocampus in the spatiotemporal organization of information. Our suggestion that NMDARs of the deep cortical layers could play a complementary role is in agreement with the hypothesis of a role of the neocortex in path selection.

\section{References}

Arleo A, Rondi-Reig L (2006) Multimodal sensory integration and concurrent navigation strategies for spatial cognition in real and artificial organisms. In: Spatial perception, spatial cognition (Dolins F, Mitchell R, eds). Cambridge, UK: Cambridge UP, in press.

Burgess N, Maguire EA, O'Keefe J (2002) The human hippocampus and spatial and episodic memory. Neuron 35:625-641.

Burke SN, Chawla MK, Penner MR, Crowell BE, Worley PF, Barnes CA, McNaughton BL (2005) Differential encoding of behavior and spatial context in deep and superficial layers of the neocortex. Neuron 45:667-674.

Chan KH, Morell JR, Jarrard LE, Davidson TL (2001) Reconsideration of the role of the hippocampus in learned inhibition. Behav Brain Res 119:111-130.

Compton DM (2001) Are memories for stimulus-stimulus associations or stimulus-response associations responsible for serial-pattern learning in rats? Physiol Behav 72:643-652.

Crawley JN (2000) What's wrong with my mouse? Behavioral phenotyping of transgenic and knockout mice. New York: Wiley-Liss.

de Bruin JP, Moita MP, de Brabander HM, Joosten RN (2001) Place and response learning of rats in a Morris water maze: differential effects of fimbria fornix and medial prefrontal cortex lesions. Neurobiol Learn Mem 75:164-178.
DeCoteau WE, Kesner RP (2000) A double dissociation between the rat hippocampus and medial caudoputamen in processing two forms of knowledge. Behav Neurosci 114:1096-1108.

Eichenbaum H (2001) The hippocampus and declarative memory: cognitive mechanisms and neural codes. Behav Brain Res 127:199-207.

Eichenbaum H, Dudchenko P, Wood E, Shapiro M, Tanila H (1999) The hippocampus, memory and place cells: is it spatial memory or a memory space. Neuron 23:209-226.

Eichenbaum HE, Stewart C, Morris RGM (1990) Hippocampal representation in spatial learning. J Neurosci 10:331-339.

Fenton AA, Wesierska M, Kaminsky Y, Bures J (1998) Both here and there: simultaneous expression of autonomous spatial memories in rats. Proc Natl Acad Sci USA 95:11493-11498.

Ferbinteanu J, Shapiro ML (2003) Prospective and retrospective memory coding in the hippocampus. Neuron 40:1227-1239.

Frank LM, Brown EN, Wilson M (2000) Trajectory encoding in the hippocampus and entorhinal cortex. Neuron 27:169-178.

Fukaya M, Kato A, Lovett C, Tonegawa S, Watanabe M (2003) Retention of NMDA receptor NR2 subunits in the lumen of endoplasmic reticulum in targeted NR1 knockout mice. Proc Natl Acad Sci USA 100:4855-4860.

Huerta PT, Sun LD, Wilson MA, Tonegawa S (2000) Formation of temporal memory requires NMDA receptors within CA1 pyramidal neurons. Neuron 25:473-480.

Jarrard LE (1973) The hippocampus and motivation. Psychol Bull 79:1-12. Jung MW, Qin Y, McNaughton BL, Barnes CA (1998) Firing characteristics of deep layer neurons in prefrontal cortex in rats performing spatial working memory tasks. Cereb Cortex 8:437-450.

Lee I, Kesner RP (2002) Differential contribution of NMDA receptors in hippocampal subregions to spatial working memory. Nat Neurosci 5:162-168.

Lenck-Santini PP, Save E, Poucet B (2001) Place-cell firing does not depend on the direction of turn in a Y-maze alternation task. Eur J Neurosci 13:1055-1058.

Lister RG (1987) The use of a plus-maze to measure anxiety in the mouse. Psychopharmacology 92:180-185.

Martin PD, Nishijo H, Ono T (1999) A combined electrophysiological and video data acquisition system using a single computer. J Neurosci Methods 92:169-177.

McHugh TJ, Blum KI, Tsien JZ, Tonegawa S, Wilson MA (1996) Impaired hippocampal representation of space in CA1-specific NMDAR1 knockout mice. Cell 87:1339-1349.

McNaughton N (1997) Cognitive dysfunction resulting from hippocampal hyperactivity-a possible cause of anxiety disorder? Pharmacol Biochem Behav 56:603-611.

Mehta MR, Quirk MC, Wilson MA (2000) Experience-dependent asymmetric shape of hippocampal receptive fields. Neuron 25:707-715.

Morris RG (2001) Episodic-like memory in animals: psychological criteria, neural mechanisms and the value of episodic-like tasks to investigate animal models of neurodegenerative disease. Philos Trans R Soc Lond B Biol Sci 356:1453-1465.

Morris RGM, Garrud P, Rawlins JNP, O’Keefe I (1982) Place navigation impaired in rats with hippocampal lesions. Nature 297:681-683.

Morris RGM, Anderson E, Lynch G, Baudry M (1986) Selective impairment of learning and blockade of long-term potentiation by an $N$-methyl-Daspartate receptor antagonist, AP5. Nature 319:774-776.

Nakazawa K, Quirk MC, Chitwood RA, Watanabe M, Yeckel MF, Sun LD, Kato A, Carr CA, Johnston D, Wilson MA, Tonegawa S (2002) Requirement for hippocampal CA3 NMDA receptors in associative memory recall. Science 297:211-218.

Nakazawa K, Sun LD, Quirk MC, Rondi-Reig L, Wilson MA, Tonegawa S (2003) Hippocampal CA3 NMDA receptors are crucial for memory acquisition of one-time experience. Neuron 38:305-315.

Nakazawa K, McHugh TJ, Wilson MA, Tonegawa S (2004) NMDA receptors, place cells and hippocampal spatial memory requirement for hippocampal CA3 NMDA receptors in associative memory recall. Nat Rev Neurosci 5:361-372.

O'Keefe J, Nadel L (1978) The hippocampus as a cognitive map. Oxford: Clarendon.

Packard MG, Knowlton BJ (2002) Learning and memory functions of the basal ganglia. Annu Rev Neurosci 25:563-593.

Packard MG, McGaugh JL (1996) Inactivation of hippocampus or caudate 
nucleus with lidocaine differentially affects expression of place and response learning. Neurobiol Learn Mem 65:65-72.

Paylor R, Nguyen M, Crawley JN, Patrick J, Beaudet A, Orr-Urtreger A (1998) Alpha7 nicotinic receptor subunits are not necessary for hippocampal-dependent learning or sensorimotor gating: a behavioral characterization of Acra7-deficient mice. Learn Mem 5:302-316.

Pellow S, Chopin P, File SE, Briley M (1985) Validation of open-closed arm entries in an elevated plus maze as a measure of anxiety in the rat. J Neurosci Methods 14:149-167.

Poucet B, Lenck-Santini PP, Hok V, Save E, Banquet JP, Gaussier P, Muller RU (2004) Spatial navigation and hippocampal place cell firing: the problem of goal encoding. Rev Neurosci 15:89-107.

Rondi-Reig L, Libbey M, Eichenbaum H, Tonegawa S (2001) CA1 NMDA receptors are required for non spatial relational memory. Proc Natl Acad Sci USA 98:3543-3548.
Tracy AL, Jarrard LE, Davidson TL (2001) The hippocampus and motivation revisited: appetite and activity. Behav Brain Res 127:13-23.

Tsien JZ, Chen DF, Gerber D, Tom C, Mercer EH, Anderson DJ, Mayford M, Kandel ER, Tonegawa S (1996a) Subregion- and cell type-restricted gene knockout in mouse brain. Cell 87:1317-1326.

Tsien JZ, Huerta PT, Tonegawa S (1996b) The essential role of hippocampal CA1 NMDA receptor-dependent synaptic plasticity in spatial memory. Cell 87:1327-1338.

White NM, McDonald RJ (2002) Multiple parallel memory systems in the brain of the rat. Neurobiol Learn Mem 77:125-184.

Wiener SI (1993) Spatial and behavioral correlates of striatal neurons in rats performing a self-initiated navigation task. J Neurosci 13:3802-3817.

Wood ER, Dudchenko PA, Robitsek RJ, Eichenbaum H (2000) Hippocampal neurons encode information about different types of memory episodes occurring in the same location. Neuron 27:623-633. 